\title{
Optimizing future remnant liver prior to major hepatectomies: increasing volume while decreasing morbidity and mortality
}

\author{
Hoylan Fernandez ${ }^{1}$, Silvio Nadalin ${ }^{2}$, Giuliano Testa ${ }^{1}$ \\ ${ }^{1}$ Simmons Transplant Institute, Baylor University Medical Center, Dallas, TX, USA; ${ }^{2}$ Department of General, Visceral and Transplant Surgery, \\ Tuebingen University Hospital, Tuebingen, Germany \\ Correspondence to: Hoylan Fernandez, MD. Simmons Transplant Institute, Baylor University Medical Center, 3410 Worth Street Suite 950, Dallas, \\ TX, USA. Email: Hoylan.Fernandez@bswhealth.org. \\ Provenance and Peer Review: This article was commissioned by the Editorial Office, Hepatobiliary Surgery and Nutrition. The article did not undergo \\ external peer review. \\ Comment on: Panaro F, Giannone F, Riviere B, et al. Perioperative impact of liver venous deprivation compared with portal venous embolization in \\ patients undergoing right hepatectomy: preliminary results from the pioneer center. Hepatobiliary Surg Nutr 2019;8:329-37.
}

Submitted Oct 09, 2019. Accepted for publication Oct 24, 2019.

doi: $10.21037 /$ hbsn.2019.10.24

View this article at: http://dx.doi.org/10.21037/hbsn.2019.10.24

Post hepatectomy liver failure (PHLF) remains the most dreaded complication in major hepatectomies. Adequate future remnant liver (FRL) plays a pivotal role in prevention of PHLF. Pre-operative portal vein embolization (PVE) has become standard of care for increasing the FRL in preparation for major hepatectomies. Associating liver partition and portal vein ligation for staged hepatectomy (ALPPS) has also been used, though has demonstrated a substantial risk of morbidity and mortality. However, there are many situations in which PVE achieves an inadequate extent of hypertrophy, potential increasing the risk of PHLF. Panaro and colleagues explore their data with a prospective review of preoperative PVE versus liver venous deprivation (LVD) regarding intra and post-operative complications, as well as, histologic findings (1). This study adds to a growing body of literature assessing the benefit of LVD over PVE and ALPPS both, in regards to increased rate of hypertrophy, improvement in FRL, in the face of similar morbidity/mortality rates compared to PVE.

PVE has been used since described by Makuuchi et al. in 1984 as the primary technique to increase FRL prior to resections (2-6). PVE is considered preoperatively when FRL is approximately $<25 \%$ of the standard liver volume, in an effort to prevent or decrease occurrence of PHLF. Either the left or right portal vein branches are embolized, leading to a redistribution of blood flow, causing hypertrophy in the contralateral hepatic segments to $30-40 \%$ of the total liver volume over 4-6 weeks. Approximately $20 \%$ of patients undergoing PVE are not able to undergo hepatectomy due to insufficient hypertrophy. The degree of hypertrophy and growth of FRL have been correlated to the occurrence of PHLF. Reported morbidity and mortality rates have been fairly low. Resection rates post PVE vary widely, most commonly due to tumor progression. Alvarez et al. report a $16.7 \%$ morbidity rate, $0.2 \%$ mortality rate, $96 \%$ achievement of sufficient FRL, and $66 \%$ resectability with PVE in their 23-year retrospective analysis (2). Yamashita et al. reviewed 319 cases undergoing PVE prior to resection for hepatocellular carcinoma, biliary tract cancer, or colorectal liver metastases (3). They demonstrated a $7.8 \%$ morbidity rate, $0 \%$ mortality rate, $2 \%$ PHLF rate and $80 \%$ resectability after PVE. There was no difference noted in the degree of hypertrophy among the different cancer types, however those with biliary tract cancer or colorectal liver metastases were more likely to demonstrate tumor progression leading to dropout prior to hepatectomy. A comparison of NSQIP data by Bagante et al. in preoperative PVE and non PVE hepatectomy patients, with matched propensity scores, demonstrated no difference in mortality or length of stay (4). However, those with PVE were noted to have an increased risk of bile leak, infection, and PHLF. Additional multivariate and multicenter NSQIP review by Snyder et al., showed no increase in overall morbidity or mortality in PVE patients undergoing liver 
resections over non PVE patients, but revealed an increase in liver specific complications (5).

ALPPS was proposed as an alternative procedure to PVE, exhibiting a greater increase in FRL than PVE in shorter time. However, the morbidity rates have been reported between $33-58 \%$ for ALPPS, which are much higher than the $16 \%$ seen after PVE (7). Morbidity and mortality rates for ALPPS have unfortunately been quite high due to the increased risk of PHLF, and therefore has not been used routinely in the pre-operative setting. Jiao et al. conducted a randomized trial of pre-operative radiofrequency assisted ALPPS (RALPPS) versus PVE (7). RALPPS demonstrated an increase in FRL compared to PVE, and an increased number of patients undergoing eventual surgical resection. There was no difference in morbidity in these procedures, however the RALPPS group experienced one mortality.

In an effort to increase FRL while decreasing morbidity and mortality rates experienced with ALPPS, LVD techniques have been introduced, in which PVE and HV embolization are conducted simultaneously in the anticipated lobe of resection (1,8-13). Schadde et al. conducted a randomized porcine model comparing portal vein occlusion to simultaneous portal and hepatic vein occlusion, comparing rates of hepatic hypertrophy, in an effort to delineate the role of potential portoportal collaterals that may form after PVE or ligation that may blunt hypertrophy (8). This demonstrated an increased FRL and marked reduction in collaterals in those subjects undergoing simultaneous portal and hepatic vein occlusion compared to PV occlusion alone. Hwang et al. performed sequential PVE and hepatic vein embolization (HVE) in pre-operative right hepatectomy patients, demonstrating an increase in FRL of $13.3 \%$ after PVE, 28.9\% after PVE and HVE, and 117.1\% 2 weeks following right hepatectomy (9). Guiu et al. assessed LVD with concurrent PVE and HVE in 10 patients, with FRL increase of $64.3 \%$ by 3 weeks, with maximal FRL function at 1 week and a $53.4 \%$ FRL volume increase at 1 week. They reported no patient mortalities, $90 \%$ resection rate, and no incidences of PHLF (10). A retrospective review of 7 patients undergoing combined PVE and HVE by Le Roy et al. showed a $52.6 \%$ increase in FRL with a median of 49 days to surgery, $18 \%$ kinetic growth rate per week, $0 \%$ mortality, and $0 \%$ PHLF (12). The FRL increased from $28.2 \%$ to $40.9 \% 23$ days after LVD, with decrease at 3-4 weeks likely related to decreasing vascular congestion, and demonstration of sinusoidal dilation, necrosis/atrophy on histology (12). Esposito and colleagues conducted a literature review of combined PVE/HVE studies, comprising a total of
68 patients, with FRL increase ranging from 33-63.3\%, with a post-operative morbidity rate of $10.3 \%$, mortality of $5.1 \%$ and $0 \%$ PHLF (13).

These studies have shown quite convincingly the overwhelming effect on rapid FRL increase experienced with LVD. A large volume study had not previously been performed regarding LVD, with findings subjected to smaller case series or case reports, nor had a comparison study been performed in regards to PVE versus LVD. While previous small sample size studies demonstrated the feasibility, low morbidity and mortality, and absence of PHLF in LVD, the outcomes of intra-operative and post-operative complications rates and changes in FRL compared to PVE alone had not been shown. Panaro et al. is the first larger series prospective study conducted comparing LVD and PVE regarding clinical outcomes, FRL volume measurements, and histologic data (1). A total of 53 patients undergoing major hepatectomies were examined, however, data collection was limited to the 29 patients undergoing right hepatectomies. ALPPS is usually conducted for right trisectionectomy, and therefore comparison to ALPPS outcomes will be difficult. Sixteen patients underwent PVE, and 13 underwent LVD if an expected FRL was $<25-30 \%$ or $<35-50 \%$ with underlying liver disease. LVD was performed over PVE if both volume and function of FRL was deemed insufficient. Kinetic growth rate was $16 \mathrm{cc} /$ day after LVD, compared to $4.8 \mathrm{cc} /$ day after PVE. While the volumetric aspect was thoroughly examined, there is no description regarding functional regeneration. Intraoperative measures of bleeding, transfusions, and operative time were not statistically different. No difference in the groups was encountered with post-operative bile leak, PHLF, atrophy/ sinusoidal dilation/hemorrhage/necrosis on resection specimen. Biopsy after PVE and HVE were not obtained, but would be of benefit to define mechanism of regeneration further, and respective effects on histology of background liver and tumor. Overall, LVD demonstrated rapid FRL hypertrophy, with no difference in morbidity/mortality rates post operatively when compared to PVE alone.

Optimizing FRL has been a challenging balance of maximizing volume, complete embolization, and speed of hypertrophy, while minimizing patient morbidity and mortality (Table 1). PVE alone frequently does not achieve sufficient FRL increase, and requires 4-6 weeks to perceive a volume increase. By comparison, ALPPS flaunts a rapid and substantial degree of hepatic hypertrophy, with a higher percentage of patients achieving resectability, however, at the cost of significant morbidity and mortality rate with a 
Table 1 Pros and cons of pre-hepatectomy procedures used to maximize FRL

\begin{tabular}{|c|c|c|c|c|c|}
\hline $\begin{array}{l}\text { Pre-hepatectomy } \\
\text { procedure }\end{array}$ & $\begin{array}{c}\text { Completeness of } \\
\text { embolization }\end{array}$ & $\begin{array}{c}\text { Time to achieve } \\
\text { hypertrophy }\end{array}$ & Volume maximization & Risk of PHLF & Morbidity/mortality \\
\hline PVE & - & - & - & + & + \\
\hline LVD & $+/-$ & + & + & + & + \\
\hline
\end{tabular}

FRL, future remnant liver; PVE, portal vein embolization; ALPPS, associating liver partition and portal vein ligation for staged hepatectomy; LVD, liver venous deprivation.

PHLF rate of $30-75 \%$. LVD offers an alternative to PVE, demonstrating an improvement in FRL volume and kinetic growth rate, allowing adequate and safe FRL to be achieved in a shorter time interval. Additionally, LVD demonstrates similar morbidity and mortality rates to PVE, without the marked PHLF incidence, morbidity and mortality rates seen with ALPPS. This demonstrates significant improvement in technique, allowing for safe surgical resections without significant difference in intra or postoperative complications. While the sample size of the study conducted by Panaro et al. is not robust, it is currently the largest study available, and the only one that compares PVE to LVD directly, with available long term post-operative data. This study also included patients with cirrhosis in their analysis, which has not occurred in any prior studies.

While we look forward to the results of DRAGON1, a randomized controlled trial comparing PVE to LVD, the safety of LVD has been clearly demonstrated even in the limited studies currently available. LVD has shown superior ability to achieve adequate FRL volumes in a shorter time interval compared to PVE without the high morbidity and mortality found with ALPPS. Serious consideration should be given to the adaptation of LVD into the armamentarium of contemporary hepatobiliary surgical techniques. LVD holds the potential to expand the group of patients able to undergo R0 resections safely, while decreasing the risk of PHLF, and intra and post-operative complications. It is an interventional procedure that has the ability to promise dynamic technical development and innovation.

\section{Acknowledgments}

Funding: None.

\section{Footnote}

Conflicts of Interest: All authors have completed the ICMJE uniform disclosure form (available at https://hbsn.amegroups. com/article/view/10.21037/hbsn.2019.10.24/coif). GT serves as an unpaid editorial board member of Hepatobiliary Surgery and Nutrition. The other authors have no conflicts of interest to declare.

Ethical Statement: The authors are accountable for all aspects of the work in ensuring that questions related to the accuracy or integrity of any part of the work are appropriately investigated and resolved.

Open Access Statement: This is an Open Access article distributed in accordance with the Creative Commons Attribution-NonCommercial-NoDerivs 4.0 International License (CC BY-NC-ND 4.0), which permits the noncommercial replication and distribution of the article with the strict proviso that no changes or edits are made and the original work is properly cited (including links to both the formal publication through the relevant DOI and the license). See: https://creativecommons.org/licenses/by-ncnd/4.0/.

\section{References}

1. Panaro F, Giannone F, Riviere B, et al. Perioperative impact of liver venous deprivation compared with portal venous embolization in patients undergoing right hepatectomy: preliminary results from the pioneer center. Hepatobiliary Surg Nutr 2019;8:329-37.

2. Alvarez FA, Castaing D, Figueroa R, et al. Natural history of portal vein embolization before liver resection: a 23-year analysis of intention-to-treat results. Surgery 2018;163:1257-63.

3. Yamashita S, Sakamoto Y, Yamamoto S, et al. Efficacy of Preoperative Portal Vein Embolization Among Patients with Hepatocellular Carcinoma, Biliary Tract Cancer, and Colorectal Liver Metastases: A Comparative Study Based 
on Single-Center Experience of 319 Cases. Ann Surg Oncol 2017;24:1557-68.

4. Bagante F, Spolverato G, Gleeson E, et al. ShortTerm Outcomes of Patients Undergoing Portal Vein Embolization: an ACS-NSQIP Procedure-Targeted Hepatectomy Analysis. J Gastrointest Surg 2019. [Epub ahead of print].

5. Snyder RA, Ewing JA, Parikh AA. Preoperative Portal Vein Embolization Is Not Associated with Increased Postoperative Complications After Major Hepatectomy: a Study of the National Surgical Quality Improvement Database. J Gastrointest Surg 2019. [Epub ahead of print].

6. Shimada S, Kamiyama T, Yokoo H, et al. Hepatic hypertrophy and hemodynamics of portal venous flow after percutaneous transhepatic portal embolization. BMC Surg 2019;19:23.

7. Jiao LR, Fajardo Puerta AB, Gall TMH, et al. Rapid Induction of Liver Regeneration for Major Hepatectomy (REBIRTH): A Randomized Controlled Trial of Portal Vein Embolisation versus ALPPS Assisted with Radiofrequency. Cancers (Basel) 2019. doi: 10.3390/ cancers 11030302.

8. Schadde E, Guiu B, Deal R, et al. Simultaneous hepatic

Cite this article as: Fernandez H, Nadalin S, Testa G. Optimizing future remnant liver prior to major hepatectomies: increasing volume while decreasing morbidity and mortality. HepatoBiliary Surg Nutr 2020;9(2):215-218. doi: 10.21037/ hbsn.2019.10.24 and portal vein ligation induces rapid liver hypertrophy: A study in pigs. Surgery 2019;165:525-33.

9. Hwang S, Ha TY, Ko GY, et al. Preoperative Sequential Portal and Hepatic Vein Embolization in Patients with Hepatobiliary Malignancy. World J Surg 2015;39:2990-8.

10. Guiu B, Chevallier P, Denys A, et al. Simultaneous transhepatic portal and hepatic vein embolization before major hepatectomy: the liver venous deprivation technique. Eur Radiol 2016;26:4259-67.

11. Guiu B, Quenet F, Escal L, et al. Extended liver venous deprivation before major hepatectomy induces marked and very rapid increase in future liver remnant function. Eur Radiol 2017;27:3343-52.

12. Le Roy B, Perrey A, Fontarensky M, et al. Combined Preoperative Portal and Hepatic Vein Embolization (Biembolization) to Improve Liver Regeneration Before Major Liver Resection: A Preliminary Report. World J Surg 2017;41:1848-56.

13. Esposito F, Lim C, Lahat E, et al. Combined hepatic and portal vein embolization as preparation for major hepatectomy: a systematic review. HPB (Oxford) 2019;21:1099-106. 